\title{
Lipid membranes with transmembrane proteins in shear flow
}

Atefeh Khoshnood, Hiroshi Noguchi, and Gerhard Gompper

Citation: The Journal of Chemical Physics 132, 025101 (2010); doi: 10.1063/1.3285269

View online: https://doi.org/10.1063/1.3285269

View Table of Contents: http://aip.scitation.org/toc/jcp/132/2

Published by the American Institute of Physics

\section{Articles you may be interested in}

Spontaneous curvature of bilayer membranes from molecular simulations: Asymmetric lipid densities and asymmetric adsorption

The Journal of Chemical Physics 142, 054101 (2015); 10.1063/1.4906149

Structure formation of surfactant membranes under shear flow

The Journal of Chemical Physics 139, 014702 (2013); 10.1063/1.4811239

The intermediate scattering function for lipid bilayer membranes: From nanometers to microns

The Journal of Chemical Physics 135, 194701 (2011); 10.1063/1.3657857

Computer simulations of bilayer membranes: Self-assembly and interfacial tension

The Journal of Chemical Physics 108, 7397 (1998); 10.1063/1.476160

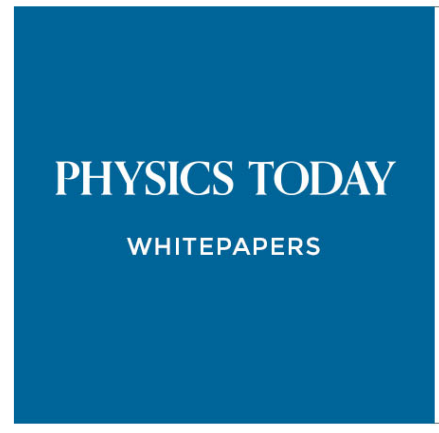

ADVANCED LIGHT CURE ADHESIVES

Take a closer look at what these environmentally friendly adhesive systems can do 


\title{
Lipid membranes with transmembrane proteins in shear flow
}

\author{
Atefeh Khoshnood, ${ }^{1,2, a)}$ Hiroshi Noguchi, ${ }^{2,3, b)}$ and Gerhard Gompper ${ }^{2,4, c)}$ \\ ${ }^{1}$ Department of Mechanical Engineering, Computational Mechanics Laboratory, \\ Sharif University of Technology, Azadi Avenue, Tehran, Iran \\ ${ }^{2}$ Institut für Festkörperforschung, Forschungszentrum Jülich, 52425 Jülich, Germany \\ ${ }^{3}$ Institute for Solid State Physics, University of Tokyo, Chiba 277-8581, Japan \\ ${ }^{4}$ Institute for Advanced Simulations, Forschungszentrum Jülich, D-52425 Jülich, Germany
}

(Received 22 September 2009; accepted 14 December 2009; published online 8 January 2010)

\begin{abstract}
The effects of embedded proteins on the dynamical properties of lipid bilayer membranes are studied in shear flow. Coarse-grained molecular simulations are employed, in which lipids are modeled as short polymers consisting of hydrophilic head groups and hydrophobic tail monomers; similarly, transmembrane proteins are modeled as connected hydrophobic double- or triple-chain molecules with hydrophilic groups at both ends. In thermal equilibrium, rigid proteinlike molecules aggregate in a membrane of flexible lipids, while flexible proteins do not aggregate. In shear flow parallel to the membrane, the monolayers of lipid bilayer slide over each other. The presence of transmembrane proteins enhances the intermonolayer friction. The friction coefficient depends on the chain lengths of lipids, the membrane tension, the length of the protein, and the cluster size. It is found to increase with protein length (with positive mismatch, i.e., proteins which are longer than the membrane thickness) and protein cluster size. In flow, proteins get oriented in the flow direction to reduce friction, with large fluctuations of the orientation angle. (C) 2010 American Institute of Physics. [doi:10.1063/1.3285269]
\end{abstract}

\section{INTRODUCTION}

Biomembranes are the main building blocks of biological cells. Besides playing the role of an envelope for the cell itself, it is a major ingredient in many organelles inside the cell. These membranes are in the fluid phase, and dynamically change their shapes to achieve their functions, in particular in the endoplasmic reticulum (ER) and the Golgi complex, where lipids and proteins are transported via vesicle budding and fusion. ${ }^{1,2}$ In the Golgi complex, a new stack of cisternae (closed disk-shaped membranes) is formed at the cis face and an old trans stack is divided into vesicles at the opposite face for vesicular transport. ${ }^{3}$ The tubular network of the ER frequently reforms their network junctions. ${ }^{2,45}$ These changes of membrane shape and topology often require the monolayers of a membrane to slide across each other.

In fact, there are various other conditions that induce slippage of monolayers of biological membranes across each other. Relative motion of membrane layers occurs when the membrane is under shear, e.g., when red blood cells (RBCs) and fluid vesicles perform a tank-treading membrane rotation under flow. ${ }^{6-11}$ A sliding between leaflets can also be induced by local-curvature change, for example when an RBC is creeping through a microvessel with a smaller diameter than of the cell itself, and the RBC is squeezed into a parachute or bullet shape. ${ }^{6,12,13}$ Another example is the formation of membrane tubes of the ER by molecular motors, ${ }^{2,4,5}$ where motor proteins such as kinesin and dynein can grab the membrane

\footnotetext{
${ }^{a)}$ Electronic mail: atefeh_khoshnood@mech.sharif.edu.

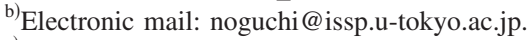

${ }^{c)}$ Electronic mail: g.gompper@fz-juelich.de.
}

and pull it as the motor itself walks along the filaments of the cytoskeleton. In in-vitro experiments, tubes are formed from a giant liposome by kinesin, ${ }^{14}$ polymerization of microtubules, ${ }^{15}$ optical tweezers, ${ }^{16}$ and hydrodynamic flow. ${ }^{17}$ Experimental tether formation by controllable force can also be employed to measure the membrane tension, ${ }^{18}$ which plays an important role in many cellular functions such as membrane trafficking and cell motility. Finally, we want to mention that many kinds of cells exhibit crawling, where the plasma membrane is under shear force. ${ }^{19}$

Biomembranes contain a large number of proteins attached to or embedded in the lipid bilayer. Here, transmembrane proteins interact with each other mediated by the membrane. When the length of the hydrophobic region of a protein has a mismatch with that of the lipid bilayer, the bilayer is locally deformed to match the lengths. This induces an attraction between the proteins in order to reduce the contact between proteins and lipids. ${ }^{20,21}$ Proteins with asymmetric shapes interact by modifying the local membrane curvature. ${ }^{2,20,21}$ Proteins with a barrel- or hourglasslike shape tilt the surrounding lipids, and this tilt induces interaction between proteins. ${ }^{21,22}$ In living cells, such membranemediated interactions can induce protein-enriched domains "rafts."

Particle-based computer simulations are a very powerful tool to study the structure and dynamics of membranes at the molecular level. Atomistic-level simulations are available only for short time dynamics and small length scales with typical current computational resources. To overcome this problem, coarse-grained descriptions have been developed, in which clusters of atoms are grouped together to form a single supersite. This significantly reduces the number of de- 
grees of freedom, but also the number of interactions to be determined. These coarse-grained lipid models are reviewed in Refs. 10 and 23-26.

Simulations of coarse-grained lipids and proteins have been employed to investigate interactions between membrane proteins. ${ }^{25,27-30}$ Typically, the proteins show strong attraction with hydrophobic mismatch and show very weak interaction without hydrophobic mismatch. In these studies, the proteins were modeled as rigid cylinders, while the flexibility and shape of proteins have not been investigated so far.

The coarse-grained model proposed by Goetz and Lipowsky ${ }^{31}$ is one of the widely used models, where the lipid and solvent molecules interacted by the Lennard-Jones (LJ) potential. Recently, Shkulipa et al. ${ }^{32}$ simulated lipid bilayer in shear flow using this LJ model, and calculated the friction coefficient of sliding between the two leaflets. They also investigated membranes of double-tail lipids with different lengths in shear flow. ${ }^{33}$ The aim of this paper is to study the behavior of protein inclusions in lipid bilayer membranes, both with and without shear flow, in particular the slipping dynamics of two monolayers induced by the flow. We simulate membranes with two different types of membrane proteins, flexible and rigid, and investigate protein tilt, protein aggregation, and protein-induced intermonolayer friction.

The theory and simulation techniques that are used for the calculation of different parameters are described in Sec. II. It contains details of the lipid and protein models and of the simulation methods. The technique for the calculation of the interlayer friction coefficient is also described. In the Results Section, we present the simulation results of three systems: membranes without protein, with flexible proteins, and with rigid proteins. The properties of a lipid bilayer membrane in the absence of proteins are studied first, followed by an analysis of the effects of the flexibility of transmembrane proteins. The main focus of the paper concerns the dependence of the intermonolayer friction coefficient on lipid tail length, membrane tension, protein length, and protein cluster size. We also investigate the tilt angle of a cluster of proteins as a function of the shear rate. Summary and discussion are given in the last section.

\section{MODELS AND METHODS}

\section{A. Lipids and proteins}

We simulate lipid membrane with embedded proteins based on the lipid model of Ref. 31. Lipid, protein, and solvent molecules are made up of two types of particles: hydrophilic and hydrophobic. Head groups of lipid and protein molecules, and also water or solvent molecules, are hydrophilic; tail groups of lipid and protein molecules are hydrophobic. Different arrangements of hydrophilic and hydrophobic particles enable us to model all molecules of interest. Some models of lipids and proteins are shown in Fig. 1. $h$ and $t$ denote head (hydrophilic) and tail (hydrophobic) particles, respectively. For example, $h t_{4}$ denotes a lipid made up of one hydrophilic and four hydrophobic particles. Other types of molecules are built by changing the number of hydrophobic particles in the chains of lipid or protein.

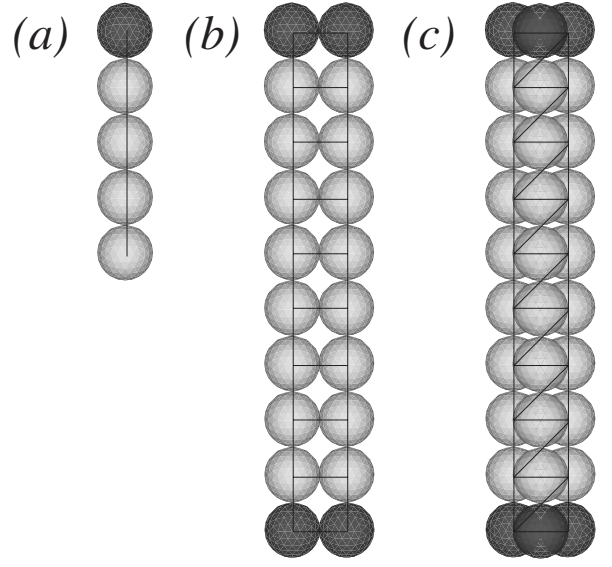

FIG. 1. Schematic representation of lipids and proteins. Black and gray circles represent hydrophilic (h) and hydrophobic (t) particles, respectively. (a) $h t_{4}$ lipid, (b) $h_{4} t_{16}$ flexible protein, and (c) $h_{6} t_{24}$ rigid protein.

The interaction between a hydrophilic and a hydrophobic particle is described by the repulsive soft-core potential,

$$
U_{\mathrm{sc}}\left(r_{i j}\right)=4 \epsilon\left(\frac{\sigma_{\mathrm{sc}}}{r_{i j}}\right)^{9},
$$

where $r_{i j}=\left|\mathbf{r}_{i}-\mathbf{r}_{j}\right|$ is the distance between the particles $i$ and $j$. Particles of the same type (either hydrophobic or hydrophilic) interacted via the LJ potential,

$$
U_{\mathrm{LJ}}\left(r_{i j}\right)=4 \epsilon\left[\left(\frac{\sigma}{r_{i j}}\right)^{12}-\left(\frac{\sigma}{r_{i j}}\right)^{6}\right] .
$$

Here, $\sigma$ and $\epsilon$ provide the length and energy scales in our simulations. The parameter $\sigma_{\mathrm{sc}}$ in Eq. (1) is chosen to be $\sigma_{\mathrm{sc}}=1.05 \sigma$. Both potentials (1) and (2) vanish in the limit $r_{i j} \rightarrow \infty$. It is therefore computationally convenient to define a cutoff radius $r_{c}=2.5 \sigma$ for both potentials. In order to form the lipid and protein molecules, we use the harmonic bond potential,

$$
U_{b}\left(r_{i, i+1}\right)=k_{b}\left(r_{i, i+1}-\sigma_{\mathrm{eq}}\right)^{2},
$$

where $r_{i, i+1}$ is the distance between two neighboring particles in a molecule. The bond stretching modulus, $k_{b}=5000 \epsilon / \sigma^{2}$, is fixed in all simulations. $\sigma_{\text {eq }}$ is the equilibrium length of a bond in lipid and protein molecules, which is chosen to be equal to $\sigma$ for bonds in both lipids and flexible proteins. Proteins are modeled as two or three identical chains, which are linked together. For flexible proteins, interchain bonds of length $\sigma_{\text {eq }}$ connect equivalent monomers [see Fig. 1(b)]. For rigid proteins, there are in addition bonds with $\sigma_{\text {eq }}=\sqrt{2} \sigma$, which connect a particle from one chain to the upper particle in the second and the lower particle of the third chain [see Fig. 1(c)].

We perform molecular dynamics (MD) simulations in the $N V T$ ensemble, in which the number of particles $N$, the volume $V$, and the temperature $T$ are held constant. LeesEdwards boundary conditions are employed to generate simple shear flow. ${ }^{34}$ The leap-frog algorithm allows the maximum time step of $\Delta t=0.005 \tau$ in the absence of shear, and $\Delta t=0.002 \tau$ when the membrane is sheared. Here, $\tau$ is the natural time scale in MD simulations with LJ potentials, 


$$
\tau=\sqrt{m \sigma^{2} / \epsilon}
$$

where $m$ is the mass of each particle. Temperature is maintained to be $k_{\mathrm{B}} T=1.35 \epsilon$ in all simulations by means of a velocity-scaling version of the profile-unbiased thermostat, ${ }^{35}$ where $k_{\mathrm{B}}$ is the Boltzmann constant. To convert dimensionless quantities into meaningful data, the values $N_{\text {avo }} \epsilon$ $=2 \mathrm{~kJ} \mathrm{~mol}^{-1}, \sigma=1 / 3 \mathrm{~nm}$, and $N_{\mathrm{avo}} m=36 \mathrm{~g} / \mathrm{mol}$ are used, where $N_{\text {avo }}$ denotes the Avogadro number. With this choice, the time scale is $\tau=1.4 \mathrm{ps}$.

A Cartesian coordinate system is placed at the center of a cubic simulation box, with the $x$-and $y$-directions parallel to the membrane plane and the $z$-direction perpendicular to it. The box sizes $L_{x}, L_{y}$, and $L_{z}$ are fixed for all simulations to be $L_{x}=L_{y}=L_{z}=18.38 \sigma$. Furthermore, the total number of particles is $N=6776$ in all simulations, which results in a fixed number density, $\rho=2 /\left(3 \sigma^{3}\right)$, and an average fluid pressure of $(1.67 \pm 0.1) \epsilon \sigma^{-3}$. The membrane is relaxed to its equilibrium state by a MD run of $100 \tau$. Friction is measured in nonequilibrium simulations, which typically extend over a time range of $(100-1000) \tau$ in each run.

We simulated three systems. Model I is a pure lipid membrane, model II is a lipid membrane with flexible proteins [see Fig. 1(b)], and model III is a membrane with rigid proteins [see Fig. 1(c)]. The normalized lipid density, $n_{\mathrm{mb}}$, is given by the number of head monomers per unit area of a monolayer, $n_{\mathrm{mb}}=N_{\mathrm{mb}} \sigma^{2} / 2 A$, measured in units of the LJ-diameter $\sigma^{2}$. For model $\mathbf{I}, N_{\mathrm{mb}}$ is the number of lipids, $N_{\text {mb }}=N_{\text {lipid }}$. A flexible or rigid protein approximately occupies the area of four or six lipids, respectively. Thus,

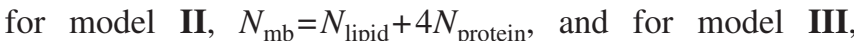
$N_{\text {mb }}=N_{\text {lipid }}+6 N_{\text {protein }}$.

\section{B. Friction coefficient}

We apply a shear flow parallel to the membrane to force the two leaflets of a membrane to slip across each other, see Fig. 2. If the simulation box is filled with a simple fluid, the velocity profile is linear with a uniform slope over the whole simulation box, $\mathbf{v}=\dot{\gamma} z \mathbf{e}_{x}$, where $\mathbf{e}_{x}$ is a unit vector in $x$ direction. However, the existence of a lipid bilayer makes the system inhomogeneous and the velocity profile becomes nonlinear, as shown in Fig. 3. Three regions can be distinguished in Fig. 3. The upper and lower regions with identical slopes represent the columns of solvent molecules above and below the membrane; they transmit the shear force to the lipid membrane, which occupies the central region of the simulation box. It is clear that the shear rate $\dot{\gamma}_{w}=d v_{x} /\left.d z\right|_{w}$ experienced by the solvent molecules is different from the overall shear rate $\dot{\gamma}$, and that it is much larger than the shear rate inside the membrane.

Figure 3 shows that there is a small difference between the velocities of the lipids in the upper and lower leaflets of the membrane. Hence, shear flow forces the layers to slide across each other. The intermonolayer friction coefficient $\xi$ is associated with the resistance of the layers to move relative to each other.

It is proportional to the shearing force $F$ and the velocity difference $\Delta v$, according to ${ }^{32}$

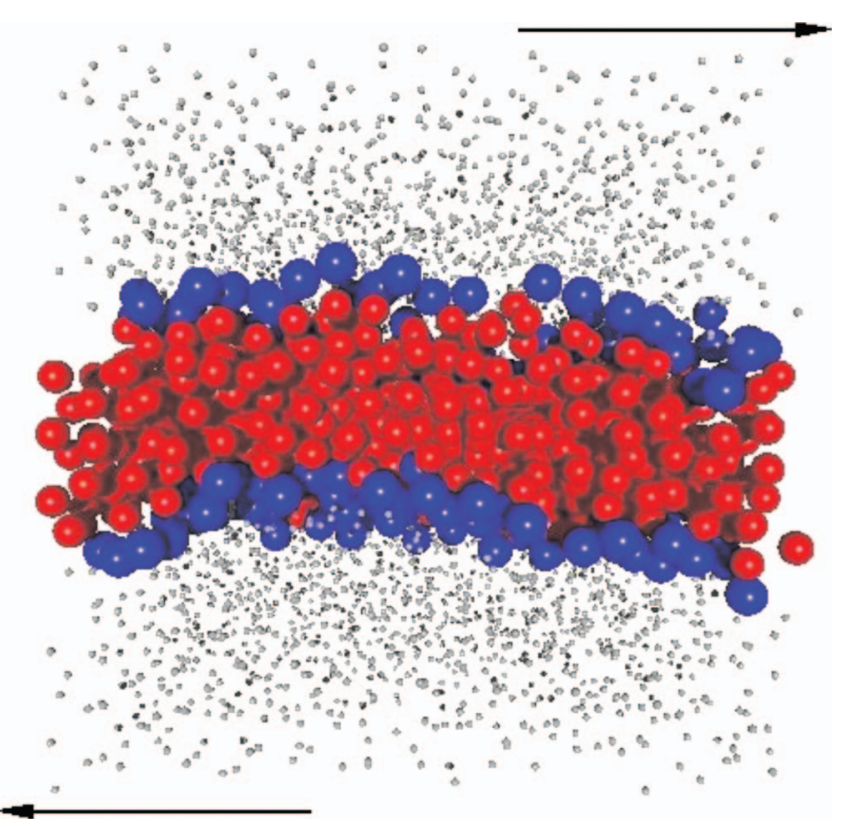

FIG. 2. Side view of the simulation box contains 288 lipid molecules of type $h t_{4}$, with arrows indicating the shearing direction. Blue and red spheres present the head and tail particles of lipids, respectively. Solvent particles are shown in gray.

$$
\xi \equiv \frac{F / A}{\Delta v},
$$

where $A$ is the projected area of the membrane, which equals the cross section $L_{x} \times L_{y}$ of the simulation box. For a sheared system, the total viscosity is defined by ${ }^{34}$

$$
\eta \equiv \frac{F / A}{\dot{\gamma}}=\frac{\left\langle P_{\alpha \beta}\right\rangle}{\dot{\gamma}},
$$

where $P_{\alpha \beta}$ are the off-diagonal elements of the pressure tensor with $\alpha=x$ representing the flow direction and $\beta=z$ representing the direction normal to the membrane plane. The operator $\langle\ldots\rangle$ denotes the canonical average. The offdiagonal components of the pressure tensor are given by ${ }^{34}$

$$
P_{\alpha \beta}=\frac{1}{V}\left\{\sum_{i=1}^{N} m_{i} v_{i \alpha} v_{i \beta}+\sum_{i=1}^{N} \sum_{j>i} r_{i j \alpha} f_{i j \beta}\right\},
$$

where $\alpha, \beta \in\{x, y, z\} . v_{i \alpha}$ and $v_{i \beta}$ are the velocity components of each particle, and $r_{i j \alpha}$ is the $\alpha$ component of the

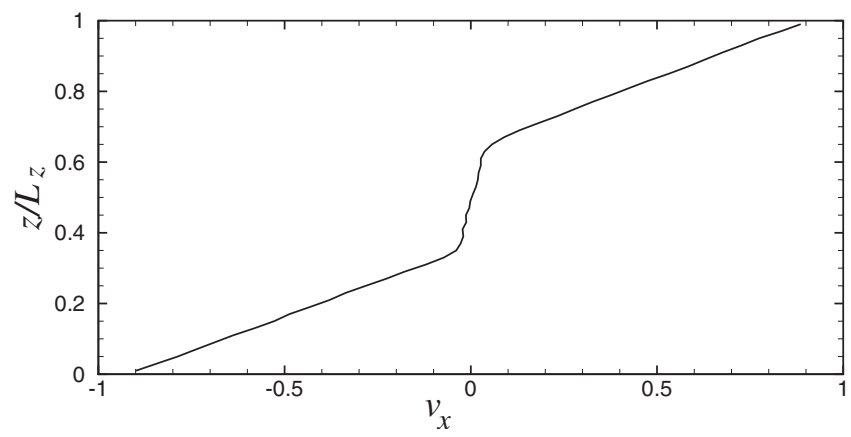

FIG. 3. The average particle velocity $v_{x}$ in the flow direction, as a function of normalized height of the simulation box, $z / L_{z}$. Simulation box contains 288 lipid molecules of type $h t_{4}$ with $\dot{\gamma}=0.1 \tau^{-1}$. 
TABLE I. Surface tension $\zeta$ of a membrane consisting of lipid $h t_{4}$ without proteins (model I), with flexible proteins (model II), and with rigid proteins (model III), with three lipid densities $n_{\mathrm{mb}}$.

\begin{tabular}{lcrcc}
\hline \hline & & \multicolumn{3}{c}{$\zeta \sigma^{2} / \epsilon$} \\
\cline { 3 - 5 } Model & $n_{\mathrm{mb}}$ & $\dot{\gamma}=0.00$ & 0.05 & 0.10 \\
\hline \multirow{3}{*}{ I } & 0.4886 & -0.570 & -0.852 & -1.503 \\
& 0.4590 & 0.034 & -0.752 & -1.441 \\
& 0.4264 & 0.088 & -0.346 & -1.060 \\
& & & & -1.012 \\
II & 0.4975 & -0.518 & -0.740 & -0.942 \\
& 0.4560 & -0.250 & -0.536 & -0.473 \\
& 0.4146 & 0.289 & 0.142 & -1.312 \\
III & 0.5004 & -0.269 & -0.294 & -0.726 \\
& 0.4619 & 0.149 & -0.228 & -0.207 \\
\hline \hline
\end{tabular}

distance between particles $i$ and $j . f_{i j \beta}$ is the $\beta$ component of the force that particle $j$ exerts on particle $i$. The average of Eq. (7) determines the numerator of Eq. (5). The slip velocity $\Delta v$ can be obtained by different methods. One possibility is to calculate $\Delta v$ from $^{32}$

$$
\Delta v=\frac{1}{2}\left[\dot{\gamma} L_{z}-\dot{\gamma}_{w}\left(L_{z}-h\right)\right],
$$

if stick boundary conditions are assumed at the membranesolvent interface. The parameter $h$ is the membrane thickness, which can be estimated from the velocity profile, e.g., by measuring the width of the central region in Fig. 3. Alternatively, the velocities of all head particles can be averaged in each leaflet of the membrane over simulation time. The velocity difference between the two layers then provides the slip velocity. The second method is obviously more precise and is used to obtain the result presented in this paper. Our results for the friction coefficient are displayed below in dimensionless form, with $\hat{\xi}=\xi \sigma^{4} / \epsilon \tau$. With the parameters given in the previous section, $\hat{\xi}=1$ corresponds to $\xi=3.8$ $\times 10^{5} \mathrm{~J} \mathrm{~s} / \mathrm{m}^{4}$.

The surface tension is another important property of a membrane. It is controlled by the number of lipids and proteins in a membrane, in a simulation box with fixed lateral extension. We compute the surface tension from ${ }^{34}$

$$
\zeta=\left[P_{z z}-\left(P_{x x}+P_{y y}\right) / 2\right] L_{z} .
$$

Here, the diagonal elements of the pressure tensor are given by

$$
P_{\alpha \alpha}=\frac{1}{V}\left(N k_{\mathrm{B}} T+\sum_{i=1}^{N} \sum_{j>i} r_{i j \alpha} f_{i j \alpha}\right),
$$

with $\alpha \in\{x, y, z\}$.

\section{RESULTS}

\section{A. Lipid bilayer without proteins}

We consider first the properties of a bilayer membrane composed of lipids $h t_{4}$ in the absence of proteins (model I). We use three lipid densities for almost tensionless, compressed, and stretched membranes. Our results are displayed in Table I. In the equilibrium simulations at $\dot{\gamma}=0$, the surface tension increases as $n_{\mathrm{mb}}$ decreases. Also, the thickness of the membrane decreases and the membrane becomes flattened with decreasing $n_{\mathrm{mb}}$. These results are in good agreement with those of previous simulations. ${ }^{31,36,37}$

The friction coefficient decreases almost linearly as the lipid density $n_{\mathrm{mb}}$ increases, as shown in Fig. 4(a). Thus, the slippage of the two leaflets of a compressed membrane over each other is easier than of a stretched membrane. If we take a look at the velocity profile of two systems of different lipid density and the same shear rate, or compares snapshots of lipid conformations, it becomes clear that the thickness of the membrane increases and forces lipid molecules to be more extended with increasing lipid density. Therefore, lipids in one layer interact less strongly with molecules in the other layer, which results in the reduction of $\xi$. The interdigitation of lipids of two layers can be illustrated by a calculation of the membrane thickness. For example, membranes in equilibrium with $n_{\mathrm{mb}}=0.4886,0.4738,0.4590$, and 0.4264 (corresponding to 330, 320, 310, and 288 lipid molecules in the simulation box) have thickness $5.65 \pm 0.08 \sigma$, $5.54 \pm 0.06 \sigma, 5.44 \pm 0.07 \sigma$, and $5.3 \pm 0.05 \sigma$, respectively. The same data as in Fig. 4(a) are plotted in Fig. 4(b) as a function of the surface tension $\zeta$. This representation shows clearly that the friction coefficient $\xi$ increases sharply with increasing tension near $\zeta=0$.

Shkulipa et al. ${ }^{32}$ used the semiflexible lipid model introduced in Ref. 31 , and found $\xi \approx 1.4 \times 10^{6} \mathrm{~J} \mathrm{~s} / \mathrm{m}^{4}$ for a tensionless membrane consisting of $h t_{4}$ lipids (a value we reproduce for the same semiflexible lipid model). For flexible $h t_{4}$ lipids, we find a somewhat larger friction coefficient $\xi$ $\approx 1.7 \times 10^{6} \mathrm{~J} \mathrm{~s} / \mathrm{m}^{4}$ for an almost tensionless membrane [compare Fig. 4(b)]. We attribute this increase in $\xi$ to the increased interdigitation of flexible chains.

When the shear rate increases from zero to $0.1 \tau^{-1}$, the tension becomes increasingly negative and the membrane experiences larger stress due to the applied shear force (see Table I). This indicates a tendency for the membrane area to increase. Indeed, for fixed surface tension, flow is expected to induce a tilt of the lipid molecules and a reduction of the membrane thickness (compare Ref. 38), which implies an increasing membrane area. This is equivalent to a negative surface tension for fixed membrane area. For fixed projected 

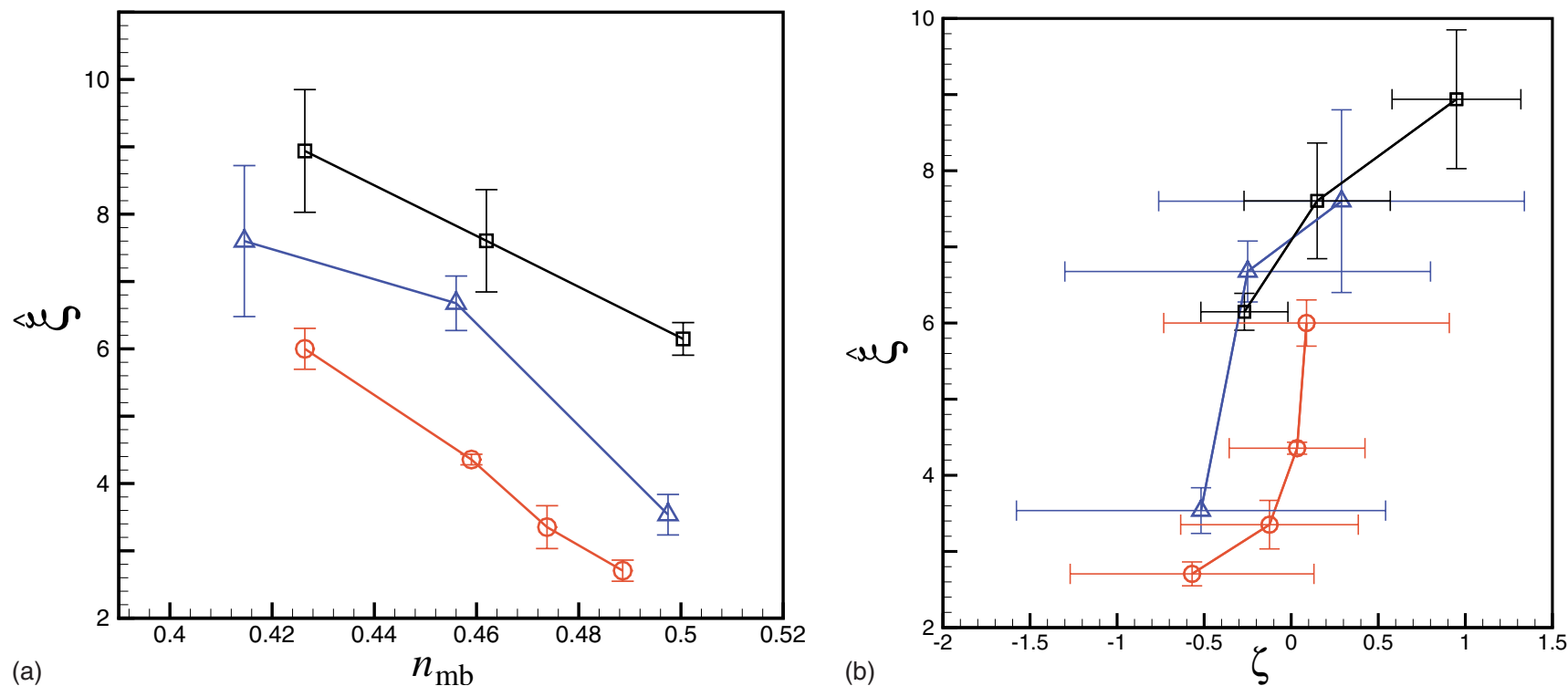

FIG. 4. Friction coefficient of a membrane, $\hat{\xi}$, as a function of (a) the number of lipids per projected area $n_{\mathrm{mb}}$ and (b) surface tension $\zeta$. Results are shown for three different systems, a membrane with no protein $(\bigcirc)$, a membrane with flexible proteins of type $h_{4} t_{16}(\triangle)$, and a membrane with rigid proteins of type $h_{6} t_{24}$ ( $\square$ ). All lipids are of the type $h t_{4} \cdot N_{\text {protein }}=8$ and $N_{\text {protein }}=9$ are used in simulations of models II and III, respectively, corresponding to a protein concentrations of about $5 \%-10 \%$. The simulations are carried out for three different shear rates $\dot{\gamma}=0.1,0.05$, and $0.03 \tau^{-1}$.

area, this could lead to a shear-induced buckling transition. Note that this behavior is in contrast to that of interfaces at two-phase coexistence in shear flow, where the surface tension has been found to increase with increasing shear rate. ${ }^{39}$

\section{B. Clustering of protein inclusions in lipid bilayers}

We investigate next the effect of flexible and rigid proteins on a lipid bilayer membrane in thermal equilibrium. Equilibrium MD simulations of two different models of proteins are performed. In both simulations, the membrane is constructed from lipids of type $h t_{4}$. The flexible and rigid proteins are of the types $h_{4} t_{16}$ and $h_{6} t_{24}$, respectively (compare Fig. 1). Proteins are initially positioned randomly on the membrane. Figure 5 shows a snapshot of the system with flexible proteins from top and bottom views. Two proteins have both of their hydrophilic ends in the same layer. This phenomenon is not unexpected since the flexibility of the proteins allows them to flip-flop and to take U-shaped conformations. Long-time simulations show that the U-shapes conformation of our flexible proteins is much more stable than the transmembrane conformation. Flexible proteins in this conformation resemble surface proteins in the cell membrane, which stay in one layer of the membrane. ${ }^{1}$ Proteins stay apart from each other during all simulations with this model.

Figure 6 shows two snapshots of the system with rigid proteins. The snapshot of Fig. 6(a) is taken at the beginning steps of simulation; it shows that three proteins are still separate, while the others have formed pairs. After more than $1500 \tau$, all proteins aggregate [see Fig. 6(b)] and make a rigid cluster, which remains stable over the subsequent simulation time. The average thickness of a tensionless bilayer of type $h t_{4}$ is $\approx 5.5 \sigma$. This is considerably smaller than the contour length of two lipids. In the case of rigid proteins, contour length and actual length are the same. Therefore, proteins
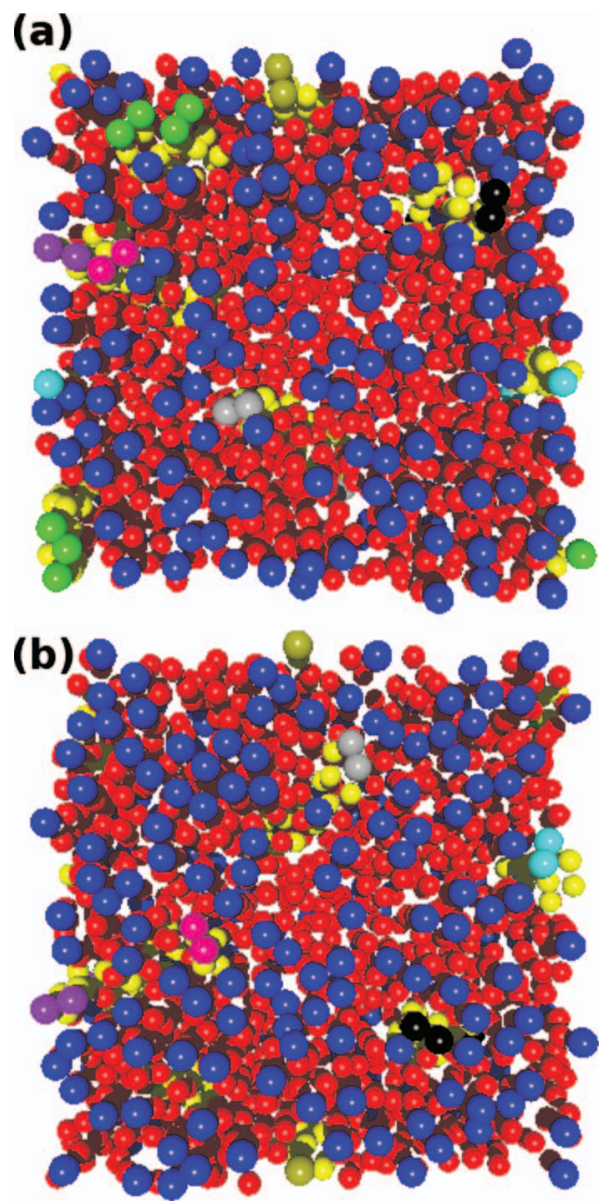

FIG. 5. Snapshot of the lipid bilayer with 248 lipid molecules of type $h t_{4}$, with eight embedded flexible proteins, $h_{4} t_{16}$, in thermal equilibrium (lipid density $n_{\mathrm{mb}}=0.415$ ). (a) Top and (b) bottom view of the membrane (at time $t=500 \tau)$. The dark blue and red spheres are lipid heads and tails, respectively. The hydrophobic particles of the proteins are yellow while other colorful particles are hydrophilic parts of the proteins, which are in the same color if they are in the same molecule. Two proteins (with green hydrophilic parts) have both of their hydrophilic ends in the bottom layer. 

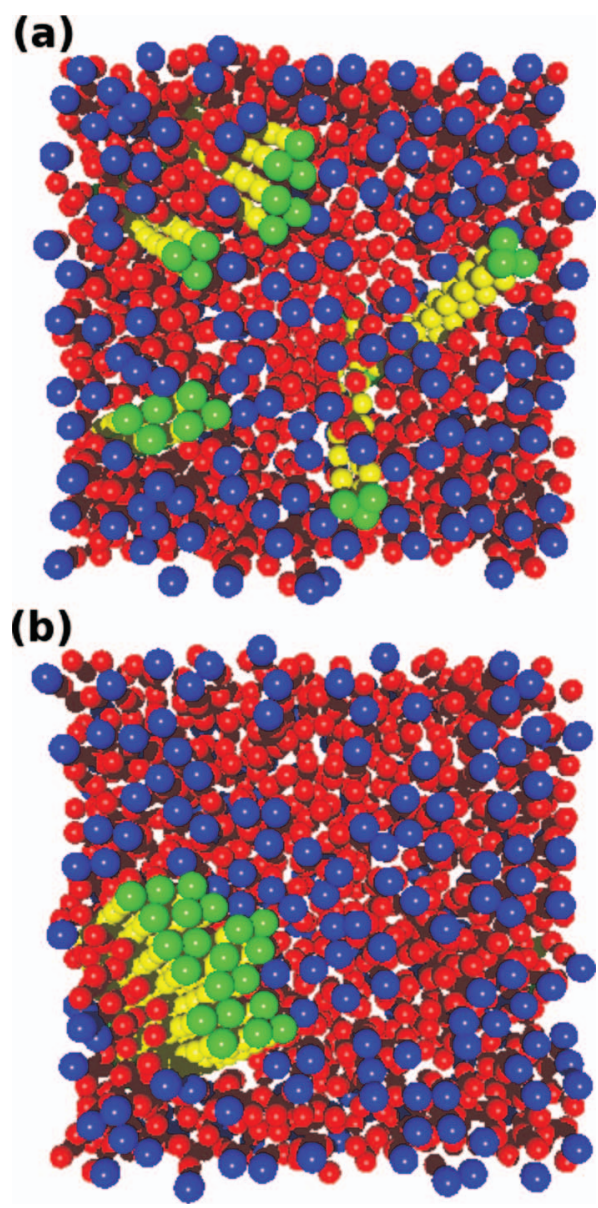

FIG. 6. Snapshots of a bilayer contain 246 lipids of type $h t_{4}$ and seven $h_{6} t_{24}$ rigid proteins in thermal equilibrium, (a) after $150 \tau$ and (b) after $1750 \tau$. The dark blue and red spheres are lipid heads and tails, respectively. The hydrophobic and hydrophilic particles of the proteins are shown by yellow and green spheres, respectively.

$h_{6} t_{24}$ have a strong positive mismatch, while proteins $h_{6} t_{15}$ have a very weak mismatch in a bilayer of the type $h t_{4}$, see Fig. 7. Our simulations show that initially separated $h_{6} t_{15}$ proteins also make stable cluster in membrane consisting of

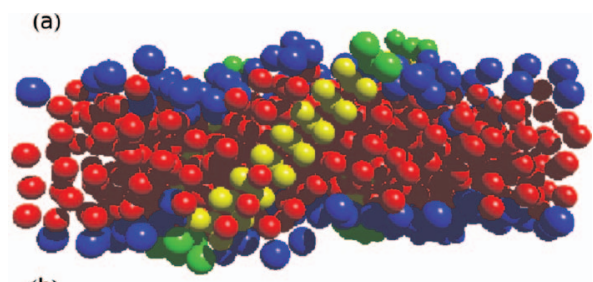

(b)

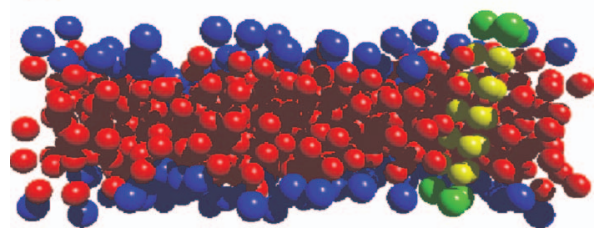

FIG. 7. Snapshots of a lipid bilayer with embedded rigid proteins, in thermal equilibrium. Side view (a) with 270 lipid molecules $h t_{4}$ and seven proteins $h_{6} t_{24}$ (after 135 ) and (b) with 280 lipid molecules $h t_{4}$ and one protein $h_{6} t_{15}$ (after $825 \tau)$. The dark blue and red spheres are lipid heads and tails, respectively. The hydrophobic particles of the proteins are yellow, the hydrophilic parts green. $h t_{4}$ lipids. Thus, protein rigidity is required for membraneinduced attractive interaction between proteins when there is no hydrophobic mismatch.

In previous simulations, ${ }^{25,27-30}$ a similar strong attraction was obtained when the length of hydrophobic protein core mismatched with the surrounding lipids, while only weak interactions were found for proteins without hydrophobic mismatch. For example, Schmidt et al. ${ }^{29}$ ascribe the clustering of transmembrane proteins to a hydrophobic mismatch, and they report a transient dimer formation in the absence of a mismatch. This is not the case in our simulations. In addition to the aggregation of proteins with positive mismatch, we have observed stable clusters even when there is no hydrophobic mismatch between lipids and proteins. Our lipid molecules are very flexible, since no bending potential is employed unlike the lipid models in previous simulations. We believe that this results into the stronger depletion interaction in our model. The depletion attraction between rigid proteins should increase with increasing lipid flexibility because the formation of protein clusters minimizes the lipidprotein contact surface and thereby reduces the total entropy.

The surface tension of the membrane does not affect the clustering. Our simulations show that aggregation of rigid proteins happens in both compressed and stretched membranes, as well as in the tensionless state. Replacing some lipids by rigid proteins increases the membrane tension, since the rigid proteins are more compact and occupy a slightly smaller area per head group. For flexible proteins, the change of the membrane tension is too small to be detected in our simulations.

\section{Lipid bilayer with protein inclusions in shear flow}

In shear flow, embedded transmembrane proteins act like pins. When the two lipid monolayers are attached locally by transmembrane proteins, their relative slipping motion is restricted. We investigate the case of small to intermediate protein concentrations, in the range of 5\%-10\%. Figure 4 shows the dependence of the friction coefficient on the lipid density, or equivalently the membrane tension. With increasing lipid density, $\xi$ decreases about linearly, both with and without proteins, see Fig. 4(a). For a fixed number of chains, the presence of rigid proteins increase the friction coefficient by about $50 \%$. The friction coefficient is less affected by flexible proteins because some flexible proteins are U-shaped and reside in a single layer. The dependence of $\xi$ on the membrane tension, shown in Fig. 4(b), reveals that the effect of membrane proteins on $\xi$ is in part due to changes in the lateral compression of the lipid molecules in the presence of proteins; for stiff proteins, the membrane compression is reduced due to the smaller entropic pressure of stiff chains, while for flexible proteins the compression is increased. In fact, stiff and flexible proteins have nearly the same effect on $\xi$ for fixed tension. The main contribution of membrane proteins on $\xi$ occurs near zero tension. Proteins shift the location of the sharp increase in $\xi$ to negative surface tension.

Figure 8 shows the dependence of $\xi$ on $n_{\mathrm{mb}}$ and $\zeta$ for longer lipids and proteins. The comparison of the results for membrane without proteins from Figs. 4(a) and 8(a) shows 

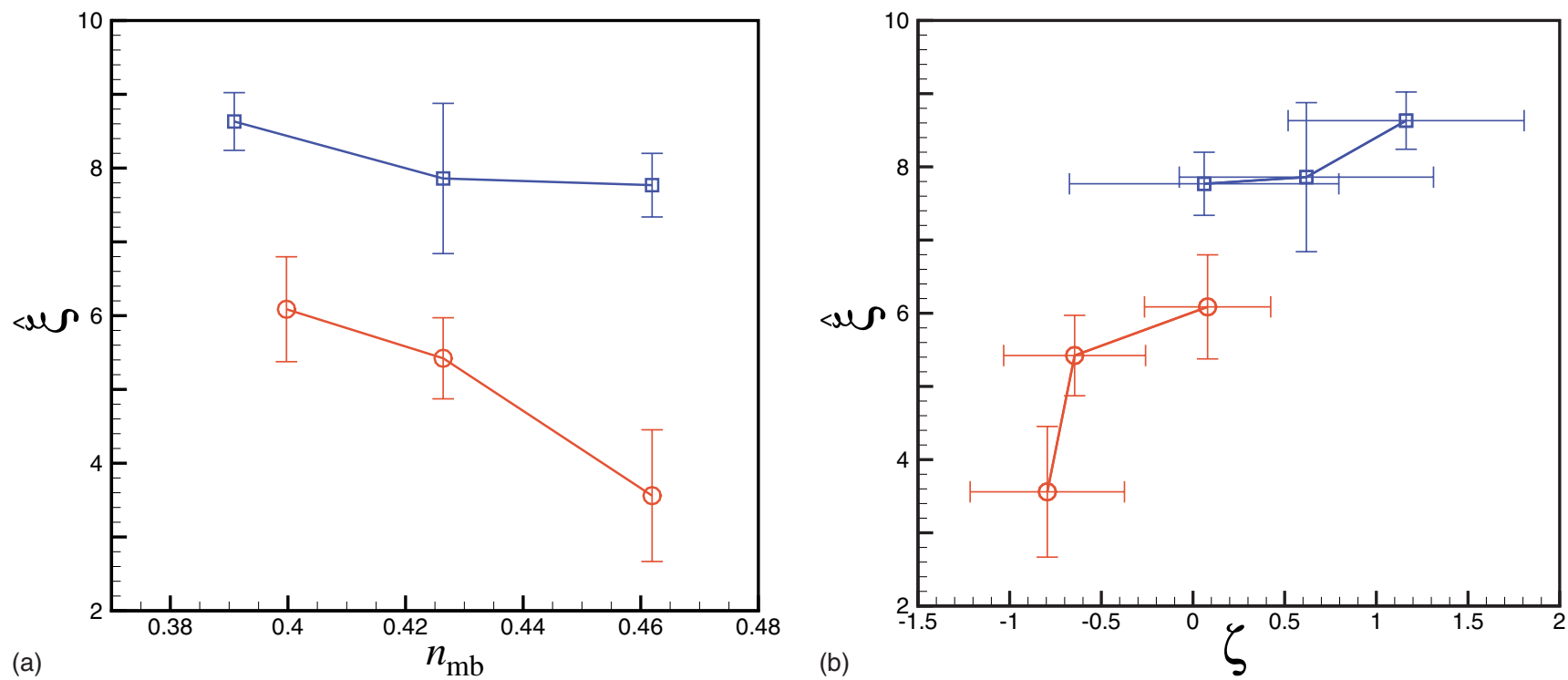

FIG. 8. Friction coefficient of a membrane, $\hat{\xi}$, as a function of (a) the number of lipids per projected area $n_{\mathrm{mb}}$ and (b) surface tension $\zeta$. Results are shown for a membrane without proteins $(\bigcirc)$ and for a membrane with rigid protein $h_{6} t_{30}(\square)$. In both cases, the membrane consists of $h t_{5}$ lipids. $N_{\text {protein }}=6$ or $N_{\text {protein }}=7$ are used in the simulations. Data are obtained from simulations with three different shear rates, $\dot{\gamma}=0.1,0.05$, and $0.03 \tau^{-1}$.

that the slopes of $\xi$ versus $n_{\mathrm{mb}}$ are almost the same for the two types of lipids $h t_{4}$ and $h t_{5}$. The friction coefficient as a function of $\zeta$ shows a sharp increase for nearly vanishing tension in both cases, see Figs. 4(b) and 8(b); however, this increase occurs at a more negative tension for longer lipids. The system of $h t_{5}$ lipids with rigid proteins $h_{6} t_{30}$ shows a weaker dependence of $\xi$ on $\zeta$, see Fig. 8(b), than in the system of $h t_{4}$ lipids with rigid proteins $h_{6} t_{24}$, see Fig. 4(b). Thus, we conclude that the friction coefficient in a membrane of longer lipids and proteins is less sensitive to the membrane tension.

This behavior can be explained by the stronger interaction of longer proteins with longer lipids, which is due to the constraint of the motion of the lipid molecules around the proteins. This implies that the mechanism of interdigitation of lipid molecules becomes less important, and the friction of the proteins within a monolayer becomes the dominant contribution. The effect of the size of lipid molecules on the intermonolayer friction coefficient has been investigated by den Otter and Shkulipa ${ }^{33}$ for single-component membranes with double-tail lipids (no proteins). Two different calculations of the intermonolayer friction coefficients were performed, in equilibrium and under shear flow. The lowest friction coefficient was found for the shortest double-tail lipid $A_{44}$ (with four hydrophobic particles per tail), a model which is structurally similar to DPPC lipids. ${ }^{40}$ In particular, the friction coefficients $\xi=2.4 \times 10^{6} \mathrm{~J} \mathrm{~s} / \mathrm{m}^{4}$ and $\xi=2.8$ $\times 10^{6} \mathrm{~J} \mathrm{~s} / \mathrm{m}^{4}$ were obtained for $A_{44}$ and $A_{55}$ lipids, respectively (in the tensionless state). ${ }^{33}$ Our results show the same trend for single-tail lipids. For example, the systems consisting of $h t_{4}$ and $h t_{5}$ lipids with almost vanishing membrane tension have the intermonolayer friction coefficients $\hat{\xi}=4.5$ and $\hat{\xi}=6.0$ (corresponding to $\xi=1.7 \times 10^{6} \mathrm{~J} \mathrm{~s} / \mathrm{m}^{4}$ and $\left.\xi=2.3 \times 10^{6} \mathrm{~J} \mathrm{~s} / \mathrm{m}^{4}\right)$, respectively.

Because rigid proteins form stable clusters during our simulations, we calculate $\xi$ for clusters of various sizes; the results are collected in Fig. 9. The simulations are performed again for compressed, almost tensionless and stretched membranes. The number of proteins in the cluster, $N_{\mathrm{P}}$, or the cross-sectional area of the cluster, is the parameter that controls the resistance of the membrane monolayers against the relative motion. The friction coefficient is found to increase roughly proportional to the cluster size $N_{\mathrm{P}}$. Except for the different offsets due to the different bare membrane frictions, the dependence of $\xi$ on $N_{\mathrm{P}}$ is approximately the same for all three membrane tensions. The larger friction forces are obtained between the monolayers of a stretched membrane, in agreement with Fig. 4(b).

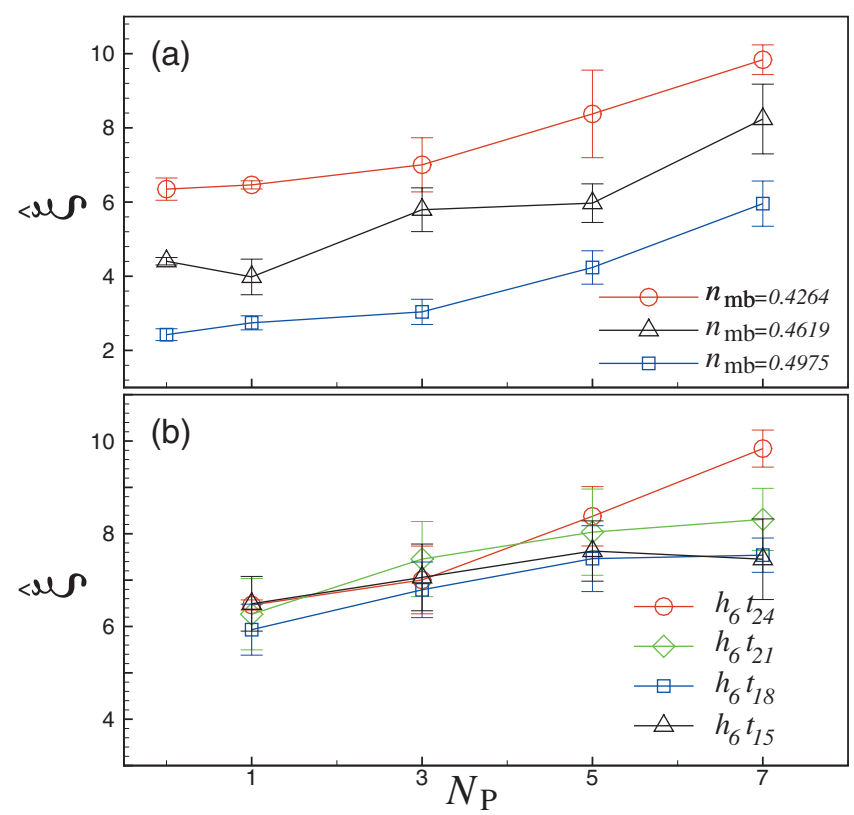

FIG. 9. The friction coefficient of lipid membrane with one embedded protein cluster, $\hat{\xi}$, as a function of number of proteins in the cluster, $N_{\mathrm{P}}$, (a) for different $n_{\mathrm{mb}}\left(\sigma^{-2}\right)$ and (b) for different types of proteins. The lipid molecules are of type $h t_{4}$. (a) Protein $h_{6} t_{24}$ (strong positive mismatch) is embedded. 
To investigate the effect of the hydrophobic mismatch on the friction coefficient, we fix the size of lipids and changed the number of monomers, i.e., the length of the hydrophobic section of the rigid proteins. Stretched membranes with lipids of type $h t_{4}$ and rigid proteins of type $h_{6} t_{24}$ (strong mismatch), $h_{6} t_{21}, h_{6} t_{18}$, and $h_{6} t_{15}$ (no mismatch), are simulated for $1000 \tau$ in equilibrium and in shear flow with $\dot{\gamma}=0.1 \tau^{-1}$. Note that all proteins except $h_{6} t_{24}$ have a smaller contour length than two lipids. The results, presented in Fig. 9(b), show that for single proteins and small clusters $\left(N_{\mathrm{P}}=3\right)$, the dependence of $\xi$ on the protein length is very small. Only for large clusters with $N_{\mathrm{P}}=7$ a clear trend emerges that (longer) proteins with a larger hydrophobic mismatch, generate a larger resistance against monolayer slippage. Since hydrophobic mismatch between lipids and protein cluster leads to lipid-induced protein tilting, and the tilt angle grows with increasing mismatch, the longest protein, $h_{6} t_{24}$, has the largest effective cross section, and thus the largest resistance against slippage.

\section{Tilt angle}

The tilt angle $\phi_{\text {tilt }}$ and the in-plane orientation $\Phi$ of the protein clusters provide insights about the conformation of transmembrane proteins in a lipid bilayer. In the previous subsection, we concluded that a larger tilt angle of proteins results in a larger interlayer friction coefficient. Therefore, we determine now directly the tilt angles of proteins both in equilibrium and under flow. In equilibrium, single proteins of type $h_{6} t_{15}, h_{6} t_{18}, h_{6} t_{21}$, and $h_{6} t_{24}$ embedded in a membrane consisting of $h t_{4}$ lipids show tilt angles of $17.6^{\circ} \pm 2.3$, $21.2^{\circ} \pm 3.3,23.3^{\circ} \pm 3.0$, and $30.8^{\circ} \pm 1.4$, respectively. This shows that larger (positive) hydrophobic mismatch results in larger tilt angles, which cause larger friction between the two layers of the membrane.

In shear flow, tilt angles are calculated for a membrane consisting of $h t_{4}$ lipids and $h_{6} t_{24}$ proteins. We focus on single proteins and on clusters with $N_{\mathrm{P}}=7$. The resulting tilt angle distributions are shown in Fig. 10. At equilibrium, the tilt angle of a cluster of $N_{\mathrm{P}}=7$ proteins is the same as for a single protein. However, for shear rates $\dot{\gamma}>0$ (in the range $\left.0.025-0.1 \tau^{-1}\right)$, the tilt angle for $N_{\mathrm{P}}=7$ is found to be larger than for $N_{\mathrm{P}}=1$. We ascribe the larger tilt angle of $N_{\mathrm{P}}=7$ protein clusters to the increased shear force on its wider hydrophilic "anchors" that make direct contact with the solvent particles.

The probability distributions of the orientation angle $\Phi$ with respect to the flow direction and the tilt angle of a cluster of $N_{\mathrm{P}}=7$ proteins under flow are shown in Fig. 11 . The location and the width of the peak of the in-plane orientation angle $\Phi$ demonstrate that the cluster is aligned with the flow direction on average, but shows large orientational fluctuations.

\section{SUMMARY AND DISCUSSION}

We have studied the dynamics of lipid membranes with embedded proteins using coarse-grained simulations. Our simulations show that rigid proteins make stable clusters even without hydrophobic mismatch. This clustering behav-

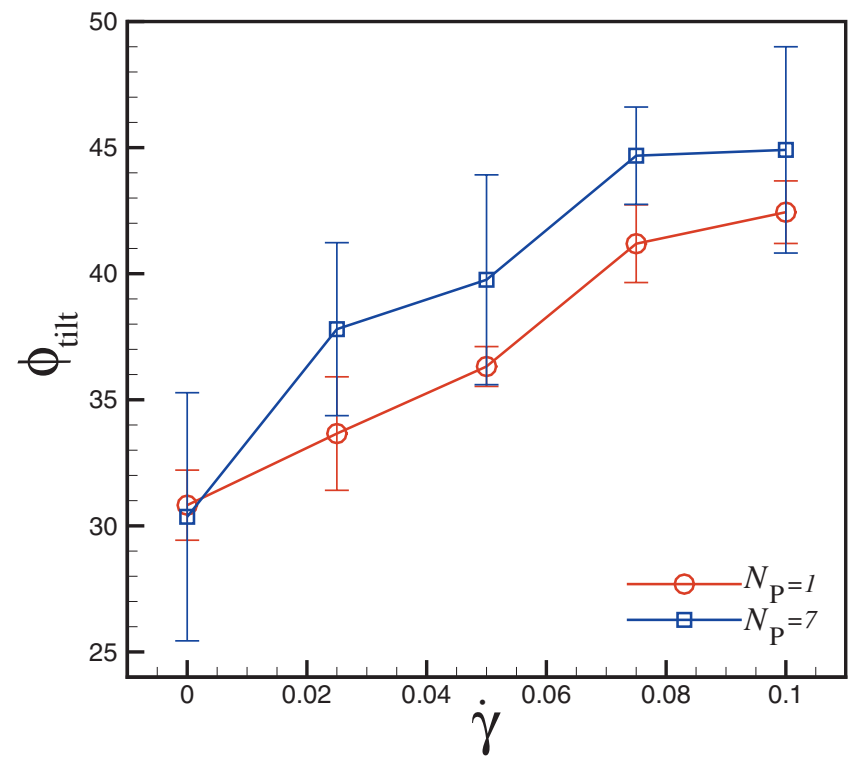

FIG. 10. Shear dependence of the tilt angle of protein clusters with one $(\bigcirc)$ and seven $(\square)$ proteins. These results are for a stretched membrane (lipid density $n_{\mathrm{mb}} \approx 0.426$ ) with $h t_{4}$ lipids and $h_{6} t_{24}$ proteins.

ior is most likely due to a strong depletion force between proteins as a result of the high flexibility of the lipid chains. Flexible proteins often have U-shaped conformations and reside in one leaflet of the membrane only; cluster formation is not observed in this case.

Transmembrane proteins enhance intermonolayer friction, since they prevent the slippage between the monolayers. Intermonolayer friction is found to increase with increasing membrane tension as well as with increasing lipid chain length. Our simulations reveal that the hydrophobic mismatch is a relevant parameter to control intermonolayer friction; longer proteins give rise to larger friction due to their

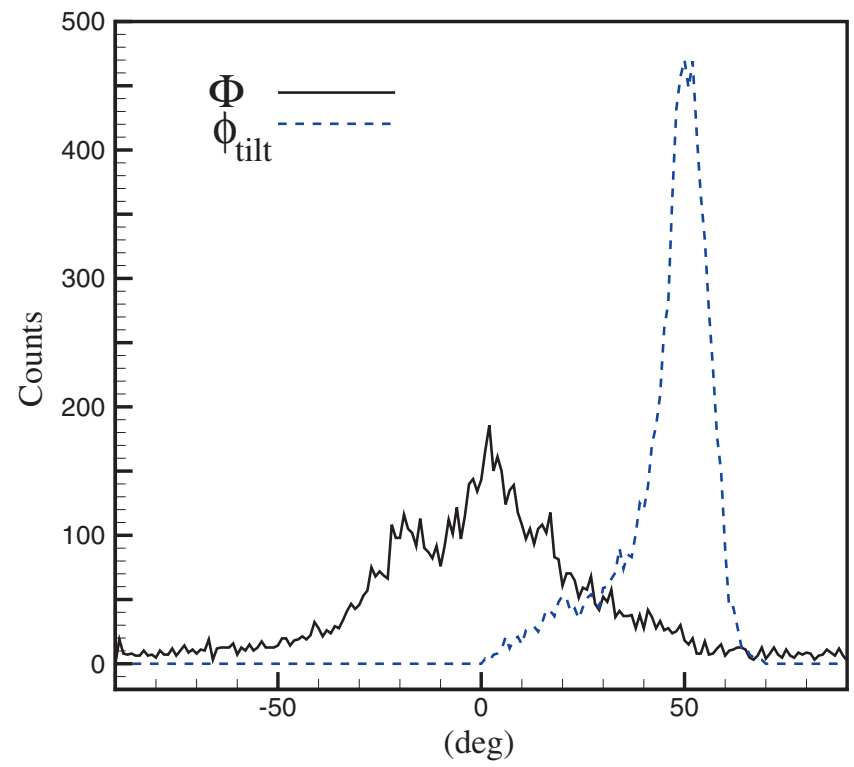

FIG. 11. Probability distribution of the orientation angle $\Phi$ with respect to the flow direction and tilt angle $\phi_{\text {tilt }}$ of cluster of $N_{\mathrm{P}}=7$ proteins of type $h_{6} t_{24}$ in a membrane of $h t_{4}$ lipids. The shear rate is $0.1 \tau^{-1}$. The distribution is sampled over a period of $16000 \tau$. In equilibrium, the average tilt angle is $\left\langle\phi_{\text {till }}\right\rangle=30.8^{\circ}$. 
larger tilt angle. Furthermore, clusters are found to have a higher resistance to tilt under shear than single proteins.

There are a number of experimental techniques for determining the intermonolayer friction coefficient $\xi$ between the two leaflets of a bilayer. One method is to apply a mechanical force, pull a bilayer through a region of high local curvature, and slide the layers over each other. This technique was used by Evans and Yeung, ${ }^{41}$ Raphael and Waugh, ${ }^{42}$ and Chizmandzhev et al. $^{43}$ Alternatively, a monolayer is fixed to a substrate and the friction coefficient is obtained by probing the diffusion of tracer lipids inside the other layer. ${ }^{44}$ Since our numerical simulations show a correlation between the intermonolayer friction coefficient and the membrane tension, future experiments should be directed toward probing such correlations. A promising experimental technique has recently been reported by Fournier et al. ${ }^{45}$ They showed that a chemically driven shape instability can trigger the ejection of a tubule, which is slowed down by intermonolayer friction. Their experiment for a mixture of egg yolk phosphatidylcholine (EYPC) and brain phosphatidylserine (PS) with mixing ratio EYPC/PS 9:1 gives $\xi \approx 2$ $\times 10^{9} \mathrm{~J} \mathrm{~s} / \mathrm{m}^{4}$ for membrane tension $\zeta \approx 10^{-7} \mathrm{~J} / \mathrm{m}^{2}$. In our simulations, $\xi \approx 1.7 \times 10^{6} \mathrm{~J} \mathrm{~s} / \mathrm{m}^{4}$ for an almost tensionless bilayer of flexible $h t_{4}$ lipids, quite similar to the vale $\xi \approx 2.4 \times 10^{6} \mathrm{~J} \mathrm{~s} / \mathrm{m}^{4}$ for a bilayer of semiflexible double-tail $A_{44}$ lipids. $^{33}$ These results are three orders of magnitude lower than the experimental value.

It is very typical that a coarse-grained model has much lower viscosity and friction than the atomistic model, since a spherical particle represents several atoms. ${ }^{23,24}$ This does not only affect the membrane, but also the solvent. In our simulation, the solvent viscosity is $1.0 \sqrt{\epsilon m} / \sigma^{2}=1.3 \times 10^{-4} \mathrm{~Pa} \mathrm{~s}$, as in Ref. 32, which is ten times smaller than the viscosity $1 \times 10^{-3} \mathrm{~Pa}$ s of water. The coarse-grained membrane simulations correctly reproduce a much higher membrane friction and viscosity than the solvent viscosity. ${ }^{32}$ However, since interdigitation and entanglements among coarse-grained lipid tails are much less pronounced than in an atomistic description, fully atomistic simulations would be needed to compare their absolute values with experiments. In any case, more data are needed before a fair comparison can be made between simulations and experiments.

Finally, we want to mention an experimental investigation of membranes with embedded proteins, which indicate protein clustering and might be used as model systems to investigate intermonolayer friction. Siebner et al. ${ }^{46}$ studied clusters of syntaxin 1 . This protein is a member of a large family of SNARE proteins, which mediate vesicle fusion in cell membrane. Siebner and colleagues applied a combination of various experimental techniques to measure the cluster size and aggregation number, and studied the mobility of syntaxin in membranes. They suggest that protein-protein interactions in the special domains of syntaxin molecules are responsible for aggregation and the constrained size of clusters. However, numerical results show that lipid-protein interactions also play a decisive role in cluster formation.

\section{ACKNOWLEDGMENTS}

A.K. greatly appreciates the hospitality of the Theoretical Soft-Matter and Biophysics group at the Forschungszentrum Jülich.

${ }^{1}$ B. Alberts, A. Johnson, J. Lewis, M. Raff, K. Roberts, and P. Walter, Molecular Biology of the Cell (Garland Science, London, UK, 2002).

${ }^{2}$ H. T. McMahon and J. L. Gallop, Nature (London) 438, 590 (2005).

${ }^{3}$ K. Matsuura-Tokita, M. Takeuchi, A. Ichihara, K. Mikuriya, and A. Nakano, Nature (London) 441, 1007 (2006).

${ }^{4}$ F. Kano, H. Kondo, A. Yamamoto, Y. Kaneko, K. Uchiyama, N. Hosokawa, K. Nagata, and M. Murata, Genes Cells 10, 989 (2005).

${ }^{5}$ M. Puhka, H. Vihinen, M. Joensuu, and E. Jokitalo, J. Cell Biol. 179, 895 (2007).

${ }^{6}$ Y. C. Fung, Biomechanics: Circulation, 2nd ed. (Springer, New York, 1997).

${ }^{7}$ M. Abkarian and A. Viallat, Soft Matter 4, 653 (2008).

${ }^{8}$ M. Kraus, W. Wintz, U. Seifert, and R. Lipowsky, Phys. Rev. Lett. 77, 3685 (1996).

${ }^{9}$ H. Noguchi and G. Gompper, Phys. Rev. Lett. 93, 258102 (2004).

${ }^{10}$ H. Noguchi, J. Phys. Soc. Jpn. 78, 041007 (2009).

${ }^{11}$ J. Deschamps, V. Kantsler, and V. Steinberg, Phys. Rev. Lett. 102, 118105 (2009).

${ }^{12}$ T. W. Secomb, R. Skalak, N. Özkaya, and J. F. Gross, J. Fluid Mech. 163, 405 (1986).

${ }^{13}$ J. L. McWhirter, H. Noguchi, and G. Gompper, Proc. Natl. Acad. Sci. U.S.A. 106, 6039 (2009).

${ }^{14}$ A. Roux, G. Cappello, J. Cartaud, J. Prost, B. Goud, and P. Bassereau, Proc. Natl. Acad. Sci. U.S.A. 99, 5394 (2002).

${ }^{15}$ H. Hotani, F. Nomura, and Y. Suzuki, Curr. Opin. Colloid Interface Sci. 4, 358 (1999).

${ }^{16}$ D. Raucher and M. P. Sheetz, Biophys. J. 77, 1992 (1999).

${ }^{17}$ O. Rossier, D. Cuvelier, N. Borghi, P. H. Puech, I. Derenyi, A. Buguin, P. Nassoy, and F. Brochard-Wyart, Langmuir 19, 575 (2003).

${ }^{18}$ J. Dai and M. P. Sheetz, Biophys. J. 77, 3363 (1999).

${ }^{19}$ D. Bray, Cell Movements: From Molecules to Motility (Garland Science, London, UK, 2000)

${ }^{20}$ R. Phillips, T. Ursell, P. Wiggins, and P. Sens, Nature (London) 459, 379 (2009).

${ }^{21}$ S. May, Curr. Opin. Colloid Interface Sci. 5, 244 (2000).

${ }^{22}$ J. B. Fournier, Eur. Phys. J. B1434-6028 11, 261 (1999).

${ }^{23}$ S. O. Nielsen, C. F. Lopez, G. Srinivas, and M. L. Klein, J. Phys.: Condens. Matter 16, R481 (2004).

${ }^{24}$ M. Müller, K. Katsov, and M. Schick, Phys. Rep. 434, 113 (2006).

${ }^{25}$ M. Venturoli, M. M. Sperotto, M. Kranenburg, and B. Smit, Phys. Rep. 437, 1 (2006).

${ }^{26}$ S. J. Marrink, A. H. de Vries, and D. P. Tieleman, Biochim. Biophys. Acta 1788, 149 (2009).

${ }^{27}$ M. Venturoli, B. Smit, and M. M. Sperotto, Biophys. J. 88, 1778 (2005).

${ }^{28}$ F. J.-M de Meyer, M. Venturoli, and B. Smit, Biophys. J. 95, 1851 (2008).

${ }^{29}$ U. Schmidt, G. Guigas, and M. Weiss, Phys. Rev. Lett. 101, 128104 (2008).

${ }^{30}$ B. West, F. L. H. Brown, and F. Schmid, Biophys. J. 96, 101 (2009).

${ }^{31}$ R. Goetz and R. Lipowsky, J. Chem. Phys. 108, 7397 (1998).

${ }^{32}$ S. A. Shkulipa, W. K. den Otter, and W. J. Briels, Biophys. J. 89, 823 (2005).

${ }^{33}$ W. K. den Otter and S. A. Shkulipa, Biophys. J. 93, 423 (2007).

${ }^{34}$ M. P. Allen and D. J. Tildesley, Computer Simulation of Liquids (Oxford University Press, Oxford, UK, 1991).

${ }^{35}$ D. J. Evans and G. P. Morriss, Phys. Rev. Lett. 56, 2172 (1986).

${ }^{36}$ R. Goetz, G. Gompper, and R. Lipowsky, Phys. Rev. Lett. 82, 221 (1999).

${ }^{37}$ W. K. den Otter, J. Chem. Phys. 123, 214906 (2005).

${ }^{38}$ D. Kaneko, T. Narita, J. P. Gong, Y. Osada, J. Ando, K. Yamamoto, S. Ohnishi, and V. V. Yaminski, J. Polym. Sci. B 41, 2808 (2003).

${ }^{39}$ D. Derks, D. G. A. L. Aarts, D. Bonn, H. N. W. Lekkerkerker, and A. Imhof, Phys. Rev. Lett. 97, 038301 (2006).

${ }^{40}$ S. J. Marrink, A. H. de Vries, and A. E. Mark, J. Phys. Chem. B 108, 750 (2004).

${ }^{41}$ E. Evans and A. Yeung, Chem. Phys. Lipids 73, 39 (1994).

${ }^{42}$ R. M. Raphael and R. E. Waugh, Biophys. J. 71, 1374 (1996). 
${ }^{43}$ Y. A. Chizmadzhev, D. A. Kumenko, P. I. Kuzmin, L. V. Chernomordik, J. Zimmerberg, and F. Cohen, Biophys. J. 76, 2951 (1999).

${ }^{44}$ R. Merkel, E. Sackmann, and E. Evans, J. Phys. (Paris) 50, 1535 (1989).

${ }^{45}$ J.-B. Fournier, N. Khalifat, N. Puff, and M. I. Angelova, Phys. Rev. Lett.
102, $018102(2009)$.

${ }^{46}$ J. J. Sieber, K. I. Willing, C. Kutzner, C. Gerding-Reimers, B. Harke, G. Donnert, B. Rammner, C. Eggeling, S. W. Hell, H. Grubmueller, and T. Lang, Science 317, 1072 (2007). 